\title{
Inhibitory Effects of Apigenin on Tumor Carcinogenesis by Altering the Gut Microbiota
}

\author{
Shichang Bian $(\mathbb{D}$, Hongjuan Wan, Xinyan Liao, and Weisheng Wang $\mathbb{D}$ \\ Tianjin Fourth Central Hospital, The Fourth Central Hospital Affiliated to Nankai University, The Fourth Central Clinical College, \\ Tianjin Medical University, Tianjin, China \\ Correspondence should be addressed to Weisheng Wang; tjdszxyygcw@126.com
}

Received 3 August 2020; Revised 11 September 2020; Accepted 19 September 2020; Published 6 October 2020

Academic Editor: Xiaolu Jin

Copyright (C) 2020 Shichang Bian et al. This is an open access article distributed under the Creative Commons Attribution License, which permits unrestricted use, distribution, and reproduction in any medium, provided the original work is properly cited.

\begin{abstract}
The flavonoid apigenin is common to many plants. Although the responsible mechanisms have yet to be elucidated, apigenin demonstrates tumor suppression in vitro and in vivo. This study uses an azoxymethane (AOM)/dextran sodium sulfate- (DSS-) induced colon cancer mouse model to investigate apigenin's potential mechanism of action exerted through its effects upon gut microbiota. The size and quantity of tumors were reduced significantly in the apigenin treatment group. Using 16S rRNA highthroughput sequencing of fecal samples, the composition of gut microbiota was significantly affected by apigenin. Further experiments in which gut microbiota were reduced and feces were transplanted provided further evidence of apigeninmodulated gut microbiota exerting antitumor effects. Apigenin was unable to reduce the number or size of tumors when gut microbiota were depleted. Moreover, tumor inhibition effects were initiated following the transplant of feces from mice treated with apigenin. Our findings suggest that the effect of apigenin on the composition of gut microbiota can suppress tumors.
\end{abstract}

\section{Introduction}

In 2012, it was estimated that there were 14.1 million new cancer cases worldwide, and by 2032, this figure is projected to increase to 25 million [1]. The incidence rate of malignant tumors was $199.4 / 10$ million in $2012 ; 8.22$ million deaths were attributed to malignant tumors, with a mortality rate of 116.3/10 million [1]. There is an urgent need to undertake more cancer prevention and treatment research to address this serious situation.

The number of symbiotic bacteria in the human gut is calculated to be approximately 100 trillion, weighing 1$2 \mathrm{~kg}$. These symbionts are made up of more than 7,000 strains representing approximately 800 bacterial genera. The microbiota can be considered an immune organism contributing to the human host's health $[2,3]$. The importance of gut flora to host health is highlighted by their critical functions of breaking down indigestible carbohydrates, inhibiting pathogenic bacterial infections, synthesizing vitamins, exerting antitumor effects, and modifying host immune reaction $[4,5]$.
Studies that compare the intestinal flora of tumor patients with that of healthy people reveal significant differences in the microbiota $[6,7]$. Gut microbiota are an important factor in treating tumors [8].

Over the past 20 years, many researchers have focused on the anticancer properties of plant-derived polyphenols [9]. Polyphenolic compounds possess one or more aromatic rings and hydroxyl functional groups and are potential anticancer drug candidates [10]. Polyphenols are naturally occurring compounds present in fruit, nuts, vegetables, and plantbased products such as spices, tea, and wine [11]. There is considerable diversity among these plant secondary metabolites, with the compounds extending from simple small molecules to complex, highly polymeric compounds [12-14]. Natural polyphenols exhibit antioxidant and antiinflammatory activities. They also have the capacity to influence molecular targets and signaling pathways, thereby modulating various processes, including angiogenesis, cell differentiation, migration, proliferation and survival, detoxification enzymes, hormone activities, and immune responses. 
It is these impressive qualities that are deemed responsible for the anticancer effects of natural polyphenols $[15,16]$.

Among other plant foods, the common flavonoid apigenin is found in oranges, onions, parsley, tea, and wheat sprouts [17]. The administration of $40-160 \mu \mathrm{M}$ of apigenin to H460 lung cancer cells resulted in damaged DNA and a concomitant increase in the production of reactive oxygen species (ROS) and $\mathrm{Ca}^{2+}[18]$. Apoptosis was induced in gastric cancer cells by apigenin $(20 \mu \mathrm{g} / \mathrm{mL})$; while normal gastric cells exhibited minimal cytotoxicity, undifferentiated gastric cancer cells were especially vulnerable [19]. Apigenin treatment $(50 \mathrm{mg} / \mathrm{kg})$ has been shown to be effective in inhibiting tumor growth and metastasis in the orthotopic colorectal cancer (CRC) model. At lower doses (20-120 $\mu \mathrm{M})$, cell proliferation, invasion, and migration have been reduced in a number of CRC cell lines [20].

The morbidity of CRC in rats fed a high-fat diet may be reduced by apigenin modulating gut microbiota; apigenin might also modulate the microbiota-gut-brain axis. The bacteria Helicobacter pylori responsible for stomach ulcers are also implicated in gastric cancer. Atrophic gastritis has been proposed as being a key precursor condition in stomach cancer induced by Helicobacter pylori. In a study of Mongolian gerbils, administering apigenin (30-60 mg/kg/week) successfully prevented the development of Helicobacter pyloriinduced atrophic gastritis and subsequent gastric cancer [21]. The in vivo mechanisms responsible for the effects of apigenin have yet to be fully characterized; however, we hypothesize that the antitumor effects of apigenin are determined by its effect upon gut microbiota.

To explore the in vivo effects of apigenin on the composition of intestinal microbiota composition, carcinogenesis, and tumors in tumor-bearing mice, this study used highthroughput sequencing of $16 \mathrm{~S}$ rRNA to refine the antitumor effects of apigenin on intestinal microbiota, and a fecal transplant experiment was conducted.

\section{Materials and Methods}

2.1. Animals. This research was approved by the animal use and welfare committee of Tianjin Fourth Central Hospital. All the experiments were performed according to the committee's animal welfare guidelines. Four-week-old femalespecific pathogen-free (SPF) BALB/c mice were acquired from the Tianjin Laboratory Animal Center (Tianjin, China). Prior to experimentation, the mice were given $5 \mathrm{~d}$ to acclimatize to the laboratory environment. All animals were kept in an air-conditioned room (temperature $23^{\circ} \mathrm{C} \pm 3^{\circ} \mathrm{C}$; relative humidity, $50 \% \pm 20 \%$; ventilated fresh air $>17$ times $/ \mathrm{h} ; 12 \mathrm{~h}$ light/dark cycle).

Dextran sulfate sodium (DSS) (MW 36-50 kDa) (Cat. No. 160110) was obtained from MP Biomedicals, LLC (Aurora, OH, USA). Mice were randomly allocated to two groups, a control group and an intervention (AP) group ( $n=8$ per group). Azoxymethane (Sigma-Aldrich) $(10 \mathrm{mg} / \mathrm{kg})$ was injected intraperitoneally to induce colon cancer. One week later, DSS (2.5\%) was added to drinking water to last for $5 \mathrm{~d}$, and then, plain drinking water was provided for the next $14 \mathrm{~d}$. This $19 \mathrm{~d}$ cycle was repeated three times. The mice in the AP group received a $30 \mathrm{mg} / \mathrm{kg}$ apigenin supplement in their diet. All mice were sacrificed 4 weeks after DSS treatment via cervical dislocation.

Cephradine (cephalosporin) and gentamicin (aminoglycoside) broad-spectrum antibiotics were administered to deplete gut flora for the commensal microbe-depleted (CMD) experiment [22]. Cefradine was prepared at a concentration of $67 \mathrm{mg} / \mathrm{kg}$, and gentamicin was prepared at a concentration of $2 \mathrm{mg} / \mathrm{kg}$ with a sterile saline solution. Antibiotics were administered by oral gavage b.i.d. for $30 \mathrm{~d}$. To evaluate the efficiency of depletion, bacterial DNA was extracted. The mice were then randomly segregated into a CMD group and a CMD-AP group ( $n=8$ per group). The same protocol was applied to the groups treated with azoxymethane (AOM) and DSS. The diet of the mice in the CMDAP group was supplemented with $30 \mathrm{mg} / \mathrm{kg}$ apigenin.

The surgical procedure for the fecal microbiota transplantation (FMT) experiment is as follows. Stools were collected from mice fed a $30 \mathrm{mg} / \mathrm{kg}$ apigenin-supplemented diet and normal SPF mice (control donor mice). The feces were collected daily under sterile conditions in laminar flow fume hoods, and the same group of feces was combined. Next, $100 \mathrm{mg}$ of feces was resuspended in $1 \mathrm{~mL}$ sterile saline and then mixed using a benchtop vortex for $10 \mathrm{~s}$. The solution was centrifuged at $800 \mathrm{~g}$ for $3 \mathrm{~min}$, and the supernatant was collected to transplant microbiota. Fresh transplant microbiota was prepared $10 \mathrm{~min}$ before use to prevent changes in bacterial composition. The mice were separated into an FMT-AP group and an FMT control group $(n=8)$. The same AOM and DSS protocol that has been described previously was applied. The FMT control group mice received $0.1 \mathrm{~mL}$ transplant microbiota from control donor mice each day, and the FMT-AP mice received $0.1 \mathrm{~mL}$ transplant material from apigenin donor mice each day [23]. Throughout the experiment, each mouse was monitored individually.

\subsection{Fecal Microbiological Determination by $16 S$ rRNA Gene} Sequencing. Prior to sacrifice, approximately $200 \mathrm{mg}$ of fecal matter was collected from each rodent. Following the manufacturer's instructions, a commercial E.Z.N.A Stool DNA Kit (Omega Bio-Tek, Inc., Norcross, GA, USA) was used to isolate the higher-quality total microbial DNA. The $20 \mu \mathrm{L}$ reaction mixture for PCR comprised $10 \mathrm{ng}$ template DNA, $1 \mu \mathrm{L}$ each of forward and reverse primers, $2 \mu \mathrm{L}$ dNTPs $(2.5 \mathrm{mM}), 4 \mu \mathrm{L}$ FastPfu buffer, and $0.5 \mu \mathrm{L}$ FastPfu polymerase; the mixture was supplemented with phosphate-buffered saline (PBS; Beyotime Biotech, Shanghai, China). The following PCR amplification process was applied: $95^{\circ} \mathrm{C}$ for $2 \mathrm{~min}$, then 30 cycles at $94^{\circ} \mathrm{C}$ for $30 \mathrm{~s}$, at $50^{\circ} \mathrm{C}$ for $30 \mathrm{~s}$, at $72^{\circ} \mathrm{C}$ for $45 \mathrm{~s}$, and at $72^{\circ} \mathrm{C}$ for $10 \mathrm{~min}$. Using a DNA gel-extracting kit (Cat. No. AP-GX250, Axygen Biosciences, Union City, CA, USA), the amplified PCR products were mixed and purified in accordance with the manufacturer's instructions. Using a DNA PCR-Free Sample Preparation Kit (Cat. No. FC-121-3003, Illumina, San Diego, CA, USA) combined with the pooled PCR products as templates, the gene library was built. To sequence the PCR products, equimolar amounts were pooled and then sequenced using the Illumina High-Throughput Sequencing Platform (mode: Hiseq2500; Illumina, San Diego, CA, USA). 


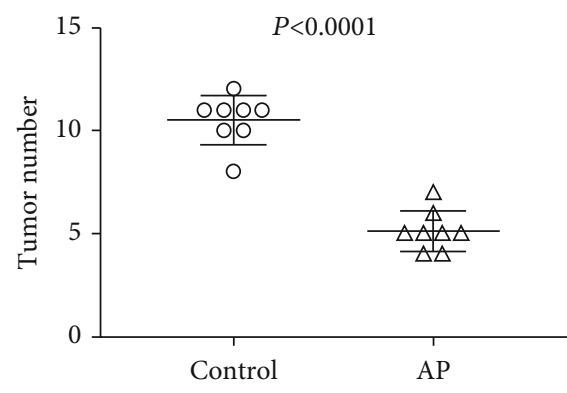

(a)

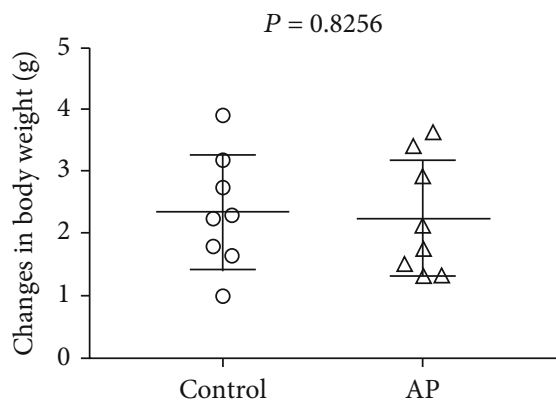

(c)

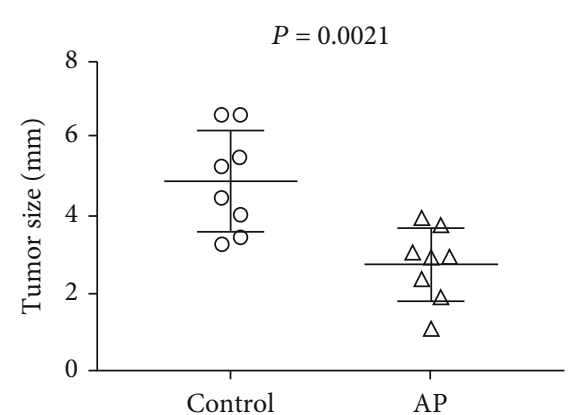

(b)

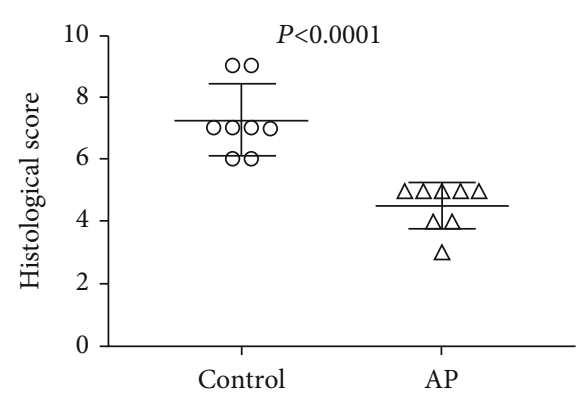

(d)

FIGURE 1: Apigenin decreased tumor morbidity in a CRC model related to colitis: the number (a) and size (b) of colon tumors in the control and AP groups, (c) change in body weight in the control and AP groups, and (d) histological score for the control and AP groups; $n=8$.

2.3. Colon Inflammatory Index. Colons and tumors were harvested for histology. Samples were processed and fixed on slides before applying hematoxylin and eosin stains. The inflammatory index was analyzed according to a previous study [24]. The inflammatory index recorded the areas of epithelial degeneration and the severity, the presence of ulcers, epithelial erosions, tissue hyperplasia, the size of the affected area, and whether the areas were focal or multifocal.

2.4. Statistical Analysis. SPSS statistical software was used to analyze the results. To assess differences involving two groups, Student's $t$-test or one-way ANOVA was used. The data are expressed as mean \pm standard deviation (SD), with $P<0.05$ indicating statistical significance.

\section{Results}

3.1. Depressor Effect of Apigenin on Tumor Growth and Metastasis In Vivo. A comparison was made of the weight and volume of tumors and the change in body weight between the AP mice and the controls (Figures 1(a)-1(c)). Although there were no changes in body weight, the control mice exhibited heavier and larger tumors $(P<0.05)$. Figure $1(\mathrm{~d})$ presents the histological scores, which are higher for the control mice $(P<0.05)$. This suggests that apigenin treatment might have helped the tumor-bearing mice to maintain some level of physical condition.

3.2. The Influence of Apigenin on Gut Microbiota in TumorBearing Mice. To investigate apigenin-altered gut microbiota, the gut bacterial populations were determined using highthroughput sequencing. Figures 2 (a) and 2(b) present the dif- ferences in the abundance of operational taxonomic units (OTUs) and the diversity index of the gut microbiota of the control and AP groups. Changes in the abundance of microbiota are illustrated in phylum-level heat maps of the community following the administration of apigenin (Figure 2(c)). There was a trend of decrease in Firmicutes (Figure 2(d)) and a significant increase in Actinobacteria (Figure 2(e)) in the AP group. Apigenin may exert antitumor effects that are responsible for changes to the phylum.

3.3. The Influence of Gut Microbiota on the Antitumor Effects of Apigenin. To examine the effect of gut microbiota on the antitumor effects of apigenin, gut microbiota were reduced using cephaloridine and gentamicin. No significant difference in the antitumor role as determined by tumor size and volume was identified between the CMD group and the CMD-AP group (Figures 3(a) and 3(b)). This indicates that after depleting the gut microbiota, apigenin did not have any benefit on the general health of the mice (Figures 3(a) and 3(b)). Furthermore, there were no significant differences in the change in body weight or histological scores (Figures 3(c) and 3(d)). However, as Figures 3(d) and 3(e) depict, the amount of OTU and bacterial 1diversity was significantly decreased in the CMD groups relative to the controls. This confirms that the antibiotics had effectively depleted gut microbiota. The relative abundance of the microbiota is shown in Figure $3(\mathrm{~g})$; we did not detect any microbial difference at the phylum level among the groups.

3.4. Regaining Antitumor Effects Using Fecal Microbiota from the Apigenin Treatment. Freshly extracted supernatant from control donor and apigenin-fed $(30 \mathrm{mg} / \mathrm{kg})$ donor mice was 


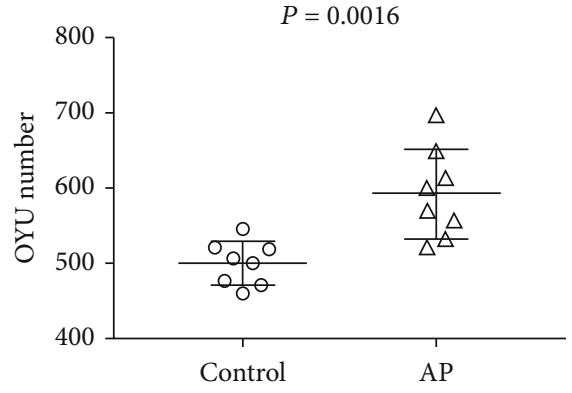

(a)

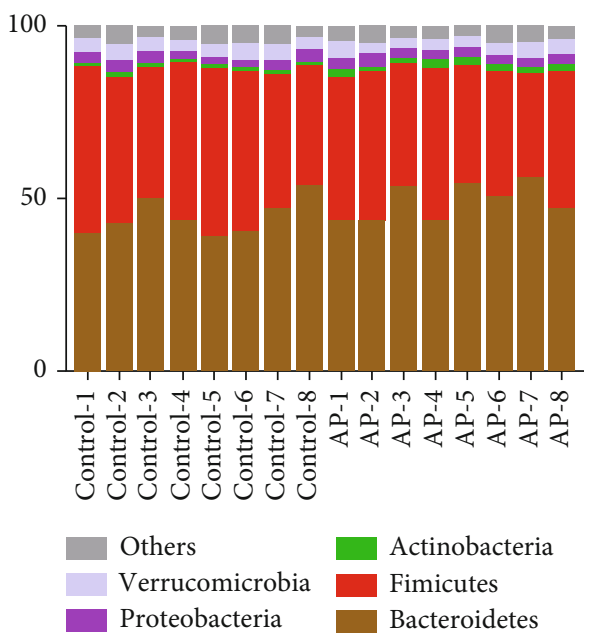

(c)

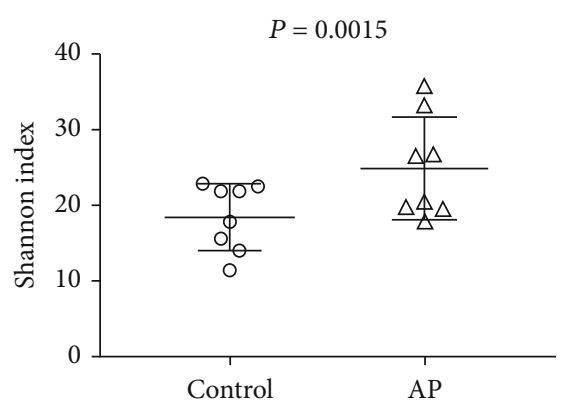

(b)

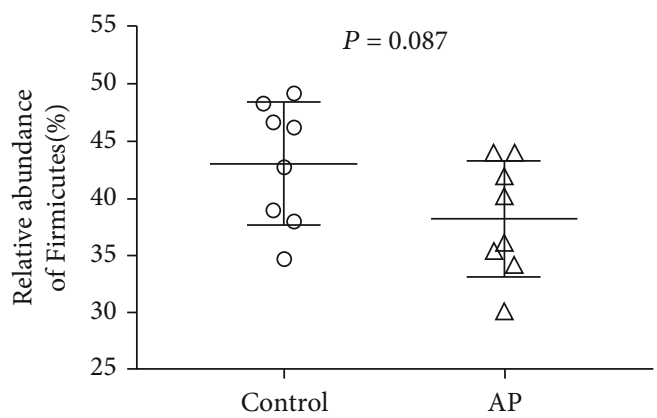

(d)

$P<0.0001$

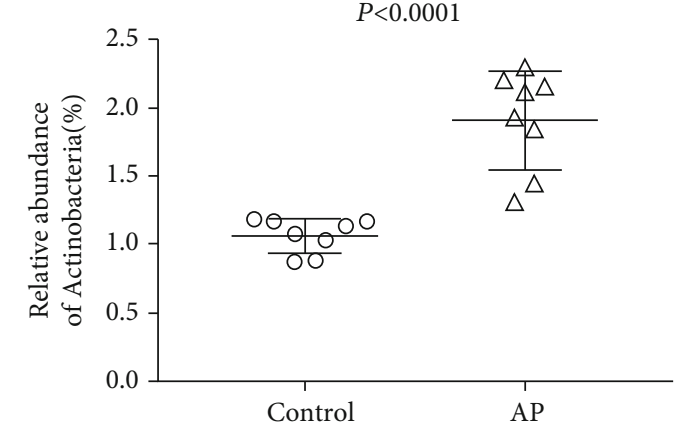

(e)

FIgURE 2: Results of 16S rRNA high-throughput sequencing: (a) OTU number and (b) Shannon index in the control ( $n=8)$ and AP $(n=8)$ groups. (c) The heat map and the relative abundance of (d) Firmicutes and (e) Actinobacteria showing differences in the abundance of gut microbiota in the different groups at the phylum level.

intragastrically administered to the FMT model and FMTAP groups over $14 \mathrm{~d}$. The number and size of tumors in the FMT group of mice were significantly greater $(P<0.05)$ than in the FMT-AP model group (Figures $4(\mathrm{a})$ and $4(\mathrm{~b})$ ), whereas no difference in the change of body weight was detected (Figure 4(c)). Similarly, the histological score of the colon in the latter group was significantly lower $(P<0.05)$ (Figure 4(d)). Meanwhile, the amount of OTU and bacterial diversity was significantly increased in the FMT-AP group relative to the control and FMT groups $(P<0.05)$ (Figures 4(e) and 4(f)). The relative abundance of the microbiota is shown in Figure $4(\mathrm{~g})$. The relative abundance of Bacteroidetes (Figure $4(\mathrm{~h})$ ) and Actinobacteria (Figure $4(\mathrm{j})$ ) in the FMT group of mice was significantly lower $(P<0.05)$ than that in the FMT-AP model group, and the relative abundance of Firmicutes (Figure 4(i)) in the FMT group of mice was significantly greater $(P<0.05)$ than that in the FMT-AP model group.

\section{Discussion}

Each year, approximately 1.2 million people are diagnosed with CRC, which is the third most prevalent cancer. There is a high incidence of CRC in developed countries $[25,26]$. There are approximately 1,000 different kinds of bacteria in the human body, amounting to $10^{14}$ individual bacteria. 


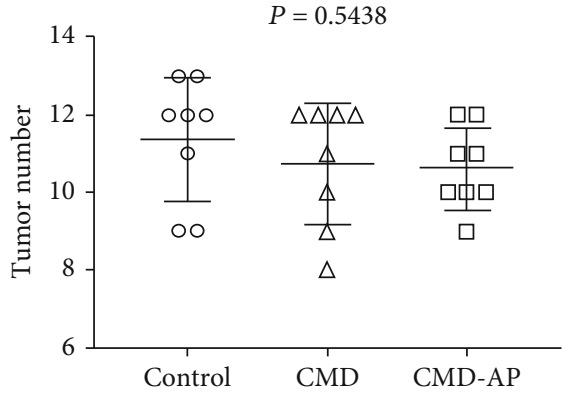

(a)

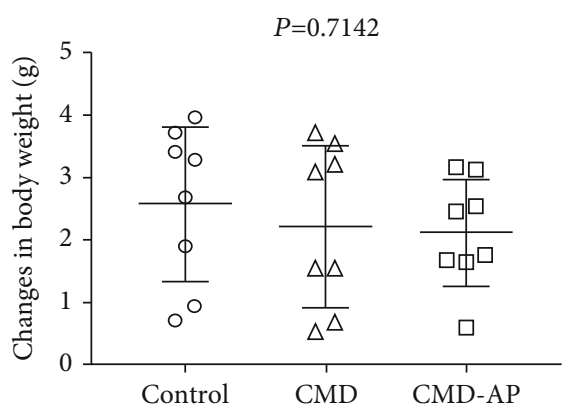

(c)

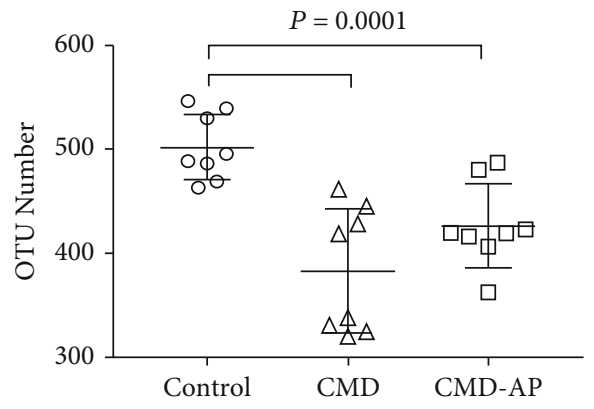

(e)

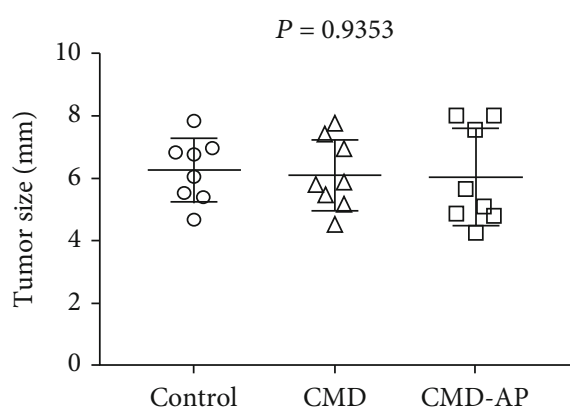

(b)

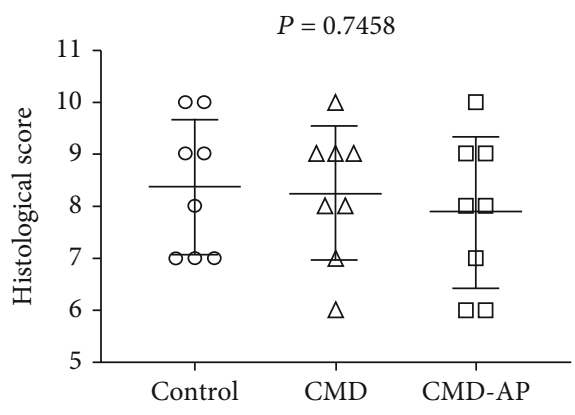

(d)

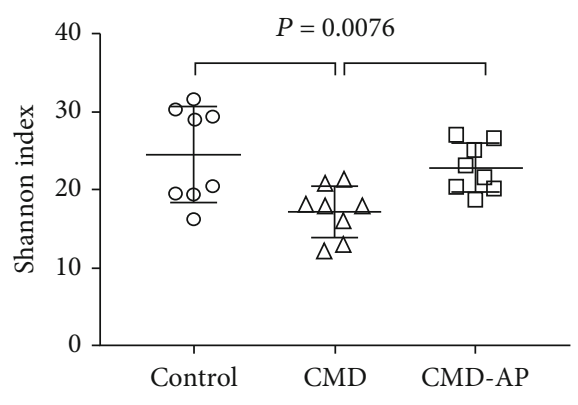

(f)

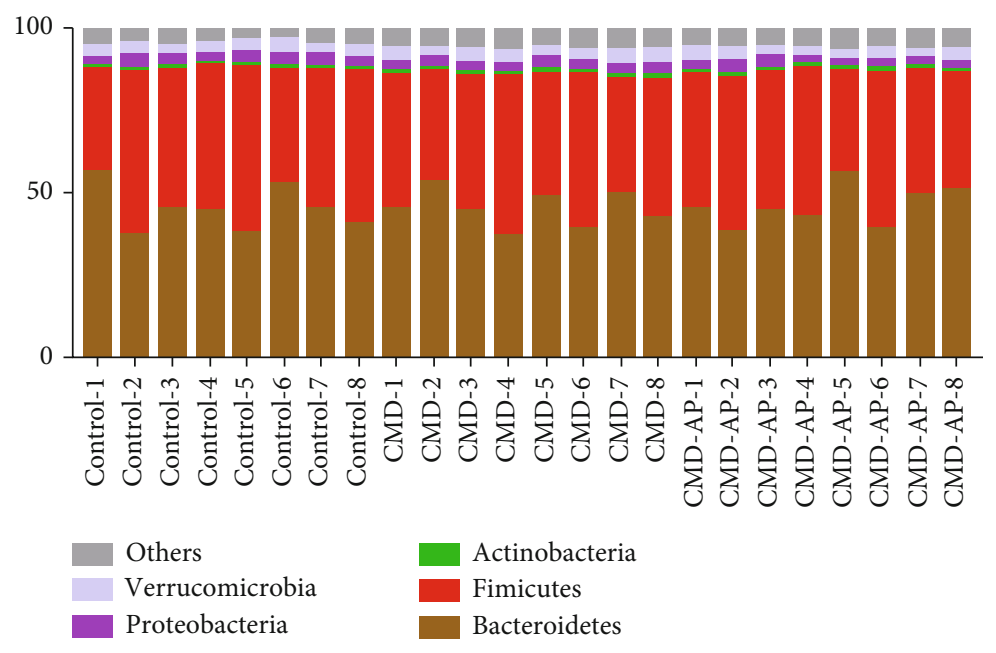

(g)

FIGURE 3: Influence of gut microbiota depletion on the antitumor effects of apigenin. The number (a) and size (b) of colon tumors in the CMD and CMD-AP groups; (c) change in body weight, (d) histological scores, (e) OTU number, (f) diversity indexes, and (g) the abundance of gut microbiota at the phylum level in the commensal microbe-depleted (CMD) experiment; $n=8$. 


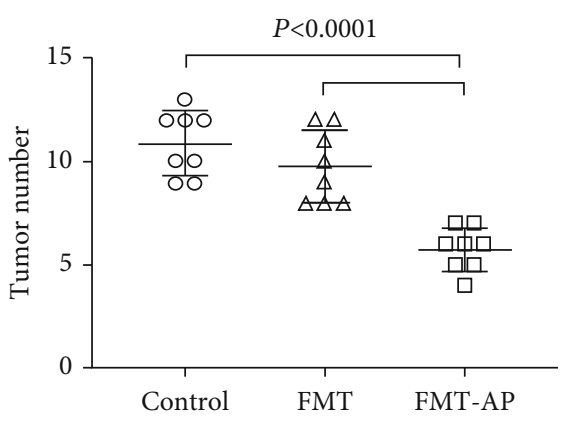

(a)

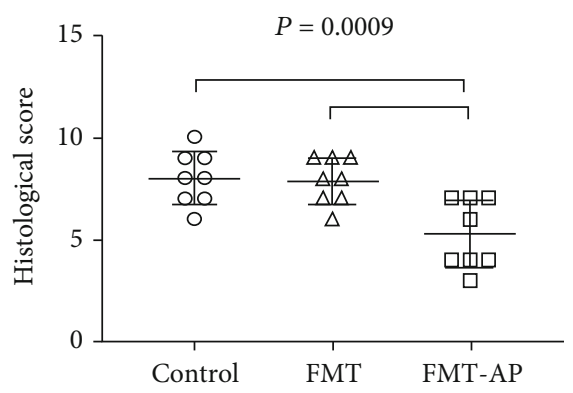

(d)

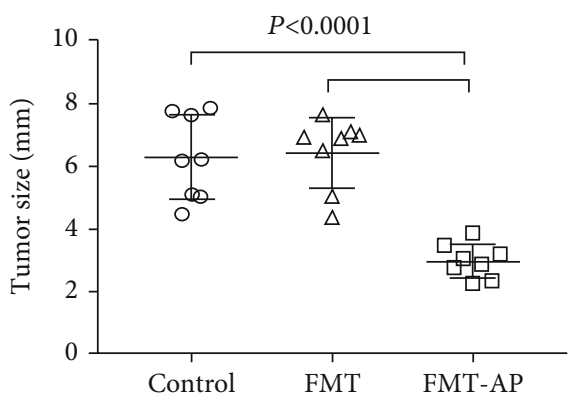

(b)

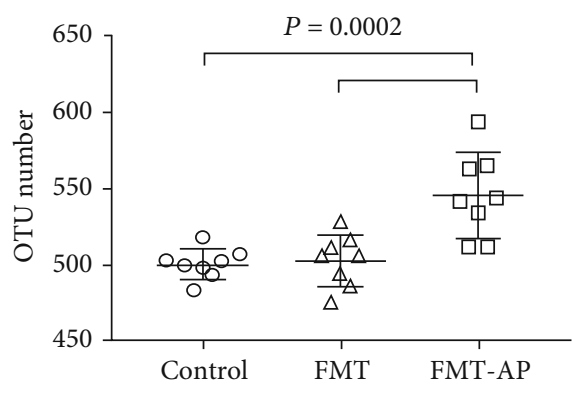

(e)

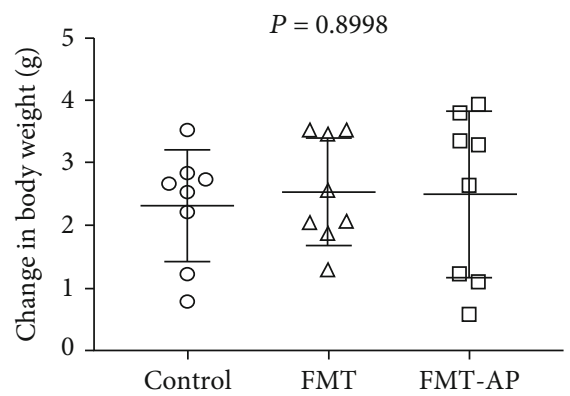

(c)

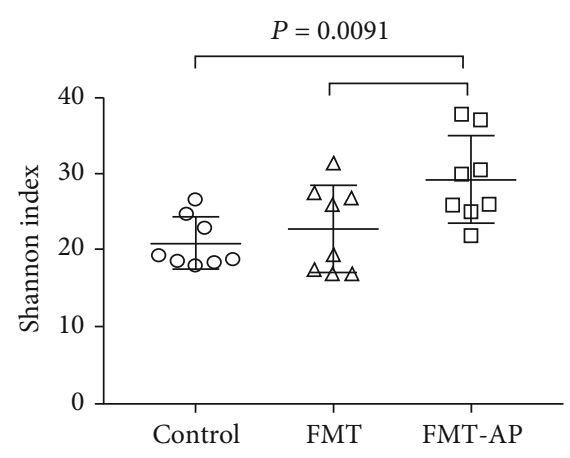

(f)

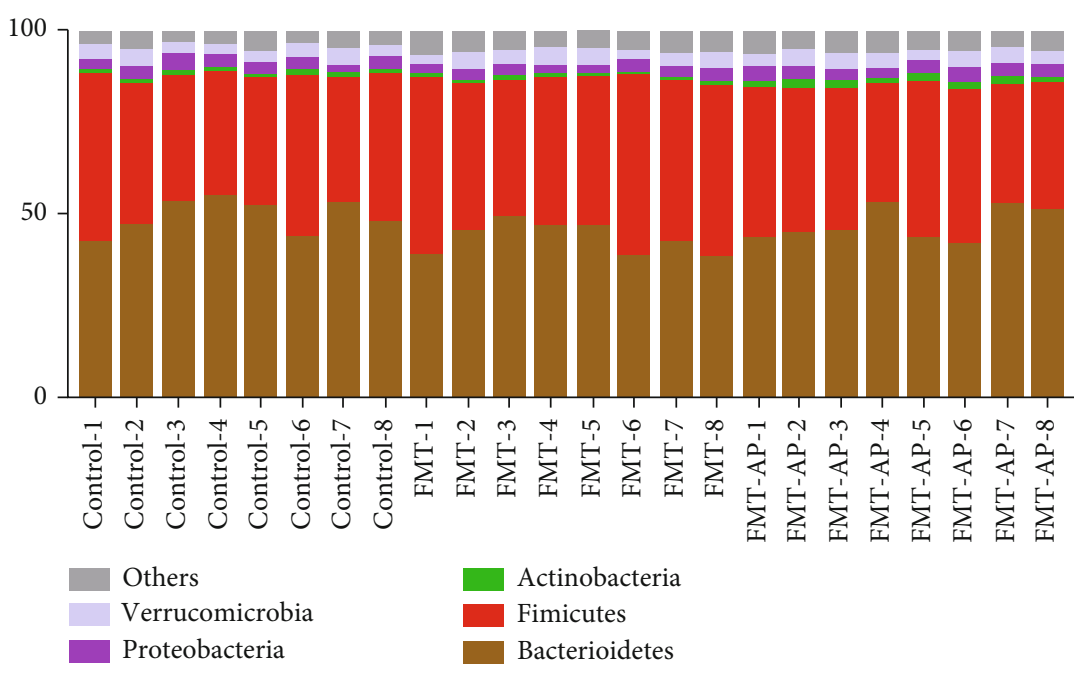

(g)

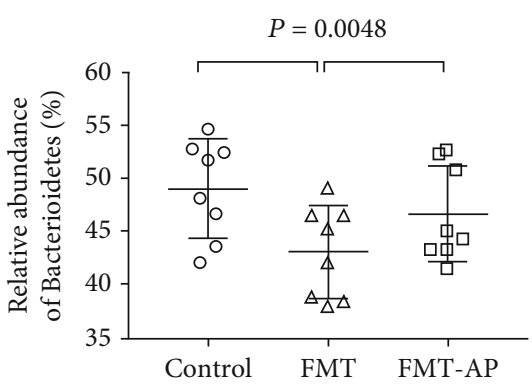

(h)

FIgure 4: Continued. 


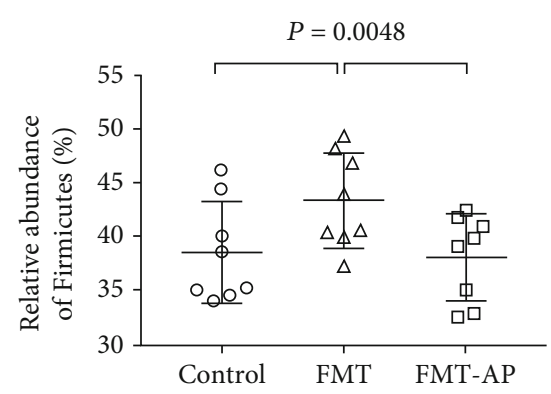

(i)

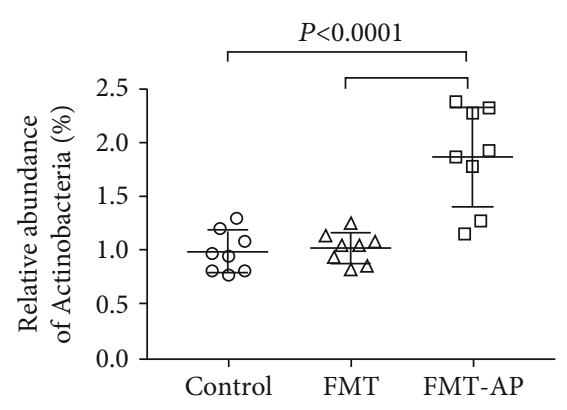

(j)

FIGURE 4: In vivo inhibition of tumor growth following fecal transplants. Number (a) and size (b) of colon tumors in the FMT ( $n=8)$ and FMT-AP ( $n=8)$ groups; (c) change in body weight, (d) histological scores, (e) OTU number, (f) diversity indexes, (g) the abundance of gut microbiota at the phylum level, and the relative abundance of (h) Bacteroidetes, (i) Firmicutes, and (j) Actinobacteria in the fecal microbiota transplantation (FMT) experiment; $n=8$.

The sum of microbial genes is calculated to be at least 150 times more than the number of human host genes [27]. Gut microbiota are symbionts, contributing to host health and to the biological barrier that aids nutrient absorption, immune regulation, and energy metabolism [28]. Studies reveal that cancer patients present with gut microbiota disorders; therefore, the rationale is that by modifying gut flora, tumor-ameliorating effects may ensue. This study administered apigenin that moderated the antitumor effects of the microbiota.

Apigenin is one of the most bioactive flavonoids and is present in many fruits and vegetables [29]. Research into apigenin started in the 1960s; by the 1980s, it was postulated that the compound had cancer-preventing properties [29, 30]. The low intrinsic toxicity of apigenin has attracted the interest of many researchers [31]. Different types of cancer and normal cell types respond differently to apigenin, influencing cancer cell growth, survival, and apoptosis [29, 30]. Although apigenin is degraded by gut microbiota, this polyphenol and its metabolites may in turn modulate the structure and function of the gut microbiota. As yet, these modulatory effects on the microbiota remain largely uncharacterized [32].

Even though there has been considerable progress in understanding cancer over the decades, there are still details relating to cancer initiation, development, metastasis, and recurrence that have yet to be elucidated. A significant challenge to acquiring a comprehensive understanding is that multiple factors are in play in tumor microenvironments. Understanding the role of the host's microbiota has been overlooked in the past but has recently become of increasing interest $[33,34]$. According to the findings of Vétizou et al., specific species of Bacteroides exert an effect upon the antitumor capacity of immune checkpoint inhibitors [35]. The effectiveness of antitumor medicine is reduced in germ-free mice with sterilized gut microbiota compared to SPF mice; when particular Bacteroides species were implanted into the intestines of mice, effectiveness was restored. Sivan et al. found that using immune checkpoint inhibitors in conjunction with a transplant of gut microbiota could significantly improve their antitumor efficacy [36].

Many symbiotic bacteria, such as Lactobacillus and Lachnospiraceae, are found in the intestines. These important species help with host digestion, maintain the intestinal environment by preventing intestinal colonization by oral bacterial clades, and exert anti-inflammatory effects. The importance of these bacteria is highlighted by studies that show microbiota dysbiosis can occur when these beneficial bacteria are absent, contributing to diabetes, inflammatory bowel disease, intestinal cancers, and obesity and adversely affecting the immune system [28, 37]. Insights into the development, metastasis, and recurrence of CRC can be increased by detailing features of dysbiosis in intestinal microbiota, especially of the large intestine. By developing a better understanding of host-microbiota interactions in CRC, the treatment of CRC patients can become more targeted. The tumor location in the intestines influences the severity of CRC [38] and the level of resistance to chemotherapy [39] in different patients. More than any other factors, diet and lifestyle are regarded as determining the probability of developing CRC. Ingesting polyphenols, such as apigenin, benefits gut microbiota and helps to protect against CRC $[40,41]$. We explored the antitumor relationship between gut microbiota and apigenin through fecal bacteria transplantation experiments. Transplanting feces from apigenin donor mice into FMT-AP mice inhibited tumor carcinogenesis in the recipient mice.

As this study has shown, in vivo tumor carcinogenesis is inhibited by apigenin by influencing the gut microbiota. However, the precise mechanism by which apigenin affects the gut microbiota and exerts its antitumor effects have yet to be determined.

\section{Data Availability}

The datasets used and/or analyzed during the current study are available from the corresponding author on reasonable request.

\section{Conflicts of Interest}

The authors declare that the research was conducted in the absence of any commercial or financial relationships that could be construed as a potential conflict of interest. 


\section{Authors' Contributions}

Shichang Bian and Hongjuan Wan contributed equally to this manuscript.

\section{Acknowledgments}

This work was supported by the National Natural Science Foundation of China (81600509) and the Tianjin Science and Technology Support Key Project Plan (20140109).

\section{References}

[1] M. Todaro, M. D'Asaro, N. Caccamo et al., "Efficient killing of human colon cancer stem cells by $\gamma \delta$ T lymphocytes," The Journal of Immunology, vol. 182, no. 11, pp. 7287-7296, 2009.

[2] W. B. Whitman, D. C. Coleman, and W. J. Wiebe, "Prokaryotes: the unseen majority," Proceedings of the National Academy of Sciences, vol. 95, no. 12, pp. 6578-6583, 1998.

[3] R. E. Ley, D. A. Peterson, and J. I. Gordon, "Ecological and evolutionary forces shaping microbial diversity in the human intestine," Cell, vol. 124, no. 4, pp. 837-848, 2006.

[4] I. Sekirov, S. L. Russell, L. C. Antunes, and B. B. Finlay, "Gut microbiota in health and disease," Physiological Reviews, vol. 90, no. 3, pp. 859-904, 2010.

[5] Q. Li, C. Ding, T. Meng et al., "Butyrate suppresses motility of colorectal cancer cells via deactivating Akt/ERK signaling in histone deacetylase dependent manner," Journal of Pharmacological Sciences, vol. 135, no. 4, pp. 148-155, 2017.

[6] T. T. Wang, G. X. Cai, Y. P. Qiu et al., "Structural segregation of gut microbiota between colorectal cancer patients and healthy volunteers," The ISME journal, vol. 6, no. 2, pp. 320329, 2012.

[7] J. R. Marchesi, B. E. Dutilh, N. Hall et al., "Towards the human colorectal cancer microbiome," PLoS One, vol. 6, no. 5, article e20447, 2011.

[8] J. Kaiser, "Gut microbes shape response to cancer immunotherapy," Science, vol. 358, no. 6363, p. 573, 2017.

[9] M. Ashrafizadeh, H. Yaribeygi, and A. Sahebkar, "Therapeutic effects of curcumin against bladder cancer: a review of possible molecular pathways," Anti-Cancer Agents in Medicinal Chemistry (Formerly Current Medicinal Chemistry-Anti-Cancer Agents), vol. 20, no. 6, pp. 667-677, 2020.

[10] C. Manach, A. Scalbert, C. Morand, C. Remesy, and L. Jimenez, "Polyphenols: food sources and bioavailability," American Journal of Clinical Nutrition, vol. 79, no. 5, pp. 727-747, 2004.

[11] S. Ding, S. Xu, J. Fang, and H. Jiang, "The protective effect of polyphenols for colorectal cancer," Frontiers in Immunology, vol. 11, p. 1407, 2020.

[12] Y. Zhou, Y. Li, T. Zhou, J. Zheng, S. Li, and H. B. Li, "Dietary natural products for prevention and treatment of liver cancer," Nutrients, vol. 8, no. 3, p. 156, 2016.

[13] L. Fu, B. T. Xu, X. R. Xu, X. S. Qin, R. Y. Gan, and H. B. Li, "Antioxidant capacities and total phenolic contents of 56 wild fruits from south China," Molecules, vol. 15, no. 12, pp. 86028617, 2010.

[14] G. F. Deng, X. Lin, X. R. Xu, L. L. Gao, J. F. Xie, and H. B. Li, "Antioxidant capacities and total phenolic contents of 56 vegetables," Journal of Functional Foods, vol. 5, no. 1, pp. 260-266, 2013.
[15] F. Li, S. Li, H. B. Li, G. F. Deng, W. H. Ling, and X. R. Xu, "Antiproliferative activities of tea and herbal infusions," Food \& Function, vol. 4, no. 4, pp. 530-538, 2013.

[16] F. Li, S. Li, H. B. Li et al., "Antiproliferative activity of peels, pulps and seeds of 61 fruits," Journal of Functional Foods, vol. 5, no. 3, pp. 1298-1309, 2012.

[17] Y. Zhu, J. Wu, S. Li et al., "Apigenin inhibits migration and invasion via modulation of epithelial mesenchymal transition in prostate cancer," Molecular Medicine Reports, vol. 11, no. 2, pp. 1004-1008, 2015.

[18] H. F. Lu, Y. J. Chie, M. S. Yang et al., "Apigenin induces apoptosis in human lung cancer H460 cells through caspaseand mitochondria-dependent pathways," Human \& Experimental Toxicology, vol. 30, no. 8, pp. 1053-1061, 2011.

[19] J. Chen, J. Chen, Z. Li, C. Liu, and L. Yin, "The apoptotic effect of apigenin on human gastric carcinoma cells through mitochondrial signal pathway," Tumor Biology, vol. 35, no. 8, pp. 7719-7726, 2014.

[20] L. Chunhua, L. Donglan, F. Xiuqiong et al., "Apigenin upregulates transgelin and inhibits invasion and migration of colorectal cancer through decreased phosphorylation of AKT," The Journal of Nutritional Biochemistry, vol. 24, no. 10 , pp. 1766-1775, 2013.

[21] C. H. Kuo, B. C. Weng, C. C. Wu, S. F. Yang, D. C. Wu, and Y. C. Wang, "Apigenin has anti-atrophic gastritis and antigastric cancer progression effects in Helicobacter pyloriinfected Mongolian gerbils," Journal of Ethnopharmacology, vol. 151, no. 3, pp. 1031-1039, 2014.

[22] Y. Zhao, J. Wu, J. V. Li, N. Y. Zhou, H. Tang, and Y. Wang, "Gut microbiota composition modifies fecal metabolic profiles in mice," Journal of Proteome Research, vol. 12, no. 6, pp. 2987-2999, 2013.

[23] C. J. Chang, C. S. Lin, C. C. Lu et al., "Ganoderma lucidum reduces obesity in mice by modulating the composition of the gut microbiota," Nature Communications, vol. 6, no. 1, 2015.

[24] H. S. Cooper, S. N. Murthy, R. S. Shah, and D. J. Sedergran, "Clinicopathologic study of dextran sulfate sodium experimental murine colitis," Laboratory Investigation; a Journal of Technical Methods and Pathology, vol. 69, no. 2, pp. 238-249, 1993.

[25] H. Brenner, M. Kloor, and C. P. Pox, "Colorectal cancer," Lancet, vol. 383, no. 9927, pp. 1490-1502, 2014.

[26] R. L. Siegel, K. D. Miller, and A. Jemal, "Colorectal cancer statistics, 2017," CA: a Cancer Journal for Clinicians, vol. 67, no. 1, pp. 7-30, 2017.

[27] MetaHIT Consortium, J. Qin, R. Li et al., "A human gut microbial gene catalogue established by metagenomic sequencing," Nature, vol. 464, no. 7285, pp. 59-65, 2010.

[28] V. Tremaroli and F. Backhed, "Functional interactions between the gut microbiota and host metabolism," Nature, vol. 489, no. 7415, pp. 242-249, 2012.

[29] S. Shukla and S. Gupta, "Apigenin: a promising molecule for cancer prevention," Pharmaceutical Research, vol. 27, no. 6, pp. 962-978, 2010.

[30] E. C. Lefort and J. Blay, "Apigenin and its impact on gastrointestinal cancers," Molecular Nutrition \& Food Research, vol. 57, no. 1, pp. 126-144, 2013.

[31] D. Tang, K. Chen, L. Huang, and J. Li, "Pharmacokinetic properties and drug interactions of apigenin, a natural flavone," Expert Opinion on Drug Metabolism \& Toxicology, vol. 13, no. 3, pp. 323-330, 2016. 
[32] F. Cardona, C. Andres-Lacueva, S. Tulipani, F. J. Tinahones, and M. I. Queipo-Ortuno, "Benefits of polyphenols on gut microbiota and implications in human health," The Journal of Nutritional Biochemistry, vol. 24, no. 8, pp. 1415-1422, 2013.

[33] X. Zhang, S. Zhao, X. Song et al., "Inhibition effect of glycyrrhiza polysaccharide (GCP) on tumor growth through regulation of the gut microbiota composition," Journal of Pharmacological Sciences, vol. 137, no. 4, pp. 324-332, 2018.

[34] G. Guan, S. Ding, Y. Yin, V. Duraipandiyan, N. A. Al-Dhabi, and G. Liu, "Macleaya cordata extract alleviated oxidative stress and altered innate immune response in mice challenged with enterotoxigenic Escherichia coli," Science China Life Sciences, vol. 62, no. 8, pp. 1019-1027, 2019.

[35] M. Vetizou, J. M. Pitt, R. Daillere et al., "Anticancer immunotherapy by CTLA-4 blockade relies on the gut microbiota," Science, vol. 350, no. 6264, pp. 1079-1084, 2015.

[36] A. Sivan, L. Corrales, N. Hubert et al., "Commensal Bifidobacterium promotes antitumor immunity and facilitates anti-PDL1 efficacy," Science, vol. 350, no. 6264, pp. 1084-1089, 2015.

[37] R. E. Ley, P. J. Turnbaugh, S. Klein, and J. I. Gordon, "Microbial ecology: human gut microbes associated with obesity," Nature, vol. 444, no. 7122, pp. 1022-1023, 2006.

[38] T. Aoyama, K. Kashiwabara, K. Oba et al., "Clinical impact of tumor location on the colon cancer survival and recurrence: analyses of pooled data from three large phase III randomized clinical trials," Cancer Medicine, vol. 6, no. 11, pp. 2523-2530, 2017.

[39] B. Flemer, D. B. Lynch, J. M. Brown et al., "Tumour-associated and non-tumour-associated microbiota in colorectal cancer," Gut, vol. 66, no. 4, pp. 633-643, 2017.

[40] S. J. O'Keefe, "Diet, microorganisms and their metabolites, and colon cancer," Nature Reviews Gastroenterology \& Hepatology, vol. 13, no. 12, pp. 691-706, 2016.

[41] M. Arnold, M. S. Sierra, M. Laversanne, I. Soerjomataram, A. Jemal, and F. Bray, "Global patterns and trends in colorectal cancer incidence and mortality," Gut, vol. 66, no. 4, pp. 683691, 2017. 